\title{
Synthesis, characterization and antimicrobial evaluation of novel diethyl (2-phenylquinazolin-4-ylamino) (phenyl) methyl phosphate
}

\author{
${ }^{1,}$ Venkateswarulu A. Maddina , ${ }^{2,}$ Mohan B. Kalyankar , ${ }^{3, P}$.A. Kulkarni \\ ${ }^{1,2,3,}$ P.G. Research Center, Department of Chemistry, Yeshwant Mahavidyalaya, Nanded-431602 (MS) India
}

\begin{abstract}
A convenient and efficient one-pot reaction has been employed for the synthesis of new aaminophosphonates $4 a-4 l$ via Kabachnik-Fields reaction in $89-95 \%$ yields. In the procedure developed, equimolar quantities of 2-phenylquinazoline-4-amine 1, various aromatic aldehydes $2 a-2 l$ and triethyl phosphite 3 was carried out under solvent-free conditions using Alum $\left(\mathrm{KAl}\left(\mathrm{SO}_{4}\right)_{2} .12 \mathrm{H}_{2} \mathrm{O}\right)$. The products were characterized by $I R,{ }^{1} \mathrm{H}$ and ${ }^{13} \mathrm{C}$ spectra and elemental analysis. All the synthesized compounds were screened for in vitro antibacterial activity (Staphylococcus aureus, Bacillus subtilis, Escherichia coli, Klebsiella pneumoniae, and Salmonella typhimurium) and antifungal activity (Candida albicans, Aspergillus niger).
\end{abstract}

KEY WORDS: $\alpha$-Aminophosphonate, Kabachnik-fields reaction, Antibacterial and antifungal activities

\section{INTRODUCTION}

Organophosphorus compounds have wide range of applications in the industrial, agricultural and medicinal chemistry owing to their unique physicochemical and biological properties. Their utility as reagents and potential synthons in organic synthesis is gaining increased attention [1]. Synthesis of aaminophosphonates, structural analogues of natural amino acids, is receiving great attention due to their applications in agriculture as plant growth regulators, herbicides [2] and virucides [3] and in medicine as anticancer agents [4], enzyme inhibitors [5], peptide mimetics [6], antibiotics and pharmacological agents [7]. As a result, a variety of synthetic approaches [8] have been developed for the synthesis of a-aminophosphonates. Of them, Kabachnik-Fields three-component reaction, in which an aldehyde, an amine and a dialkyl phosphite are reacted in one-pot set-up, is an important one. In some reports, this reaction was carried out in straight-forward one-pot procedures without any catalysts $[9,10]$. While, in most cases it was performed using catalysts, such as $\mathrm{LiClO}_{4}$ [11, 12], $\mathrm{TaCl}_{5}-\mathrm{SiO}_{2}$ [13], $\mathrm{InCl}_{3}$ [14], lanthanide-triflate [15] and $\mathrm{CF}_{3} \mathrm{COOH}$ [16]. The key step in this synthesis is the nucleophilic addition of an amine to carbonyl compound followed by addition of a trialkyl phosphite to the resulting imine. Formation of hydroxy phosphonates and product of rearrangement frequently accompany this reaction. In view of greater applicability of $\alpha$-aminophosphonates, an easy one-pot synthetic procedure for them by Kabachnik-Fields reaction under mild conditions using Alum ( $\mathrm{KAl}\left(\mathrm{SO}_{4}\right)_{2}$ as a catalyst has been developed.

\subsection{Chemistry}

\section{RESULTS AND DISCUSSION}

The synthetic route involves reaction of equimolar quantities of 2- phenylquinazoline-4-amine 1, various aromatic aldehydes 2a-2l results in the formation of imine intermediates which subsequently reacts with trialkyl phosphites using Alum (KAl $\left(\mathrm{SO}_{4}\right)_{2}$ as a catalyst to produce the corresponding $\alpha$-aminophosphonates (Scheme 1). Structurally diverse aldehydes, amines and phosphites were used to afford the corresponding $\alpha$ aminophosphonates in high to excellent yields (Table 2) herein; we would like to report that alum is an efficient catalyst for the formation of $\alpha$-aminophosphonates by one-pot three component reaction of an aldehyde, amine and triethyl phosphite under solvent-free conditions (Scheme 1).

\subsection{Microbiology}

The antimicrobial activities of these compounds were evaluated by minimum inhibitory concentration (MIC) against Gram-positive and Gram-negative test bacteria Staphylococcus aureus (MTCC 96), Bacillus subtilis (MTCC 441), Escherichia coli (MTCC 1650), Pseudomonas aeruginosa, (MTCC 1688), and fungi Aspergillus niger (MTCC 1789) and Candida albicans (MTCC 227) in vitro. Streptomycin and nystatin as reference drug, by agar diffusion method, [17-18] the results of in vitro antibacterial activities and MIC's of compound $\mathbf{4}$ a-l against various bacterial strains are summarized in Table 1, compound $\mathbf{4} \mathbf{a}, \mathbf{4} \mathbf{c}$, shows excellent activity against all bacteria with showing more than $12 \mathrm{~mm}$ zone of inhibition at $25 \mu \mathrm{g} / \mathrm{mL}$ MIC value. Compound $\mathbf{4} \mathbf{f}$ is found to be potent activity against Bacillus subtilis and Escherichia coli even at very low 
concentration where as compound $\mathbf{4} \mathbf{i}$ also point out excellent activity against Pseudomonas aeruginosa, with showing above $15 \mathrm{~mm}$ zone of inhibition even at $25 \mathrm{MIC}$ value in competition with standard drug, where as compound $4 \mathbf{f}$ is found to be almost equally potent active against Escherichia coli in comparison with streptomycin even at lowest MIC $(25 \mu \mathrm{g} / \mathrm{ml})$. Compound $\mathbf{4} \mathbf{b}, \mathbf{4} \mathbf{d}, \mathbf{4} \mathbf{e}$, and $\mathbf{4} \mathbf{~ h}$ are also found good active but at double concentration. Compound $\mathbf{4}$ a shows considerable activity against Aspergillus niger where as compound $\mathbf{4 c}$ and $\mathbf{4} \mathbf{f}$ shows good activity against both fungal species. Compound $\mathbf{4} \mathbf{i}$ found to most potent active towards Aspergillus niger and Candida albicans with showing more than $12 \mathrm{~mm}$ zone of inhibition at $25 \mu \mathrm{g} / \mathrm{mL}$ MIC value in comparison with standard drug. Likewise remaining compound $\mathbf{4 ~ b}, \mathbf{4 ~ d}, \mathbf{4 ~ e , ~} \mathbf{4} \mathrm{g}$ and, $4 \mathrm{~h}$ are also found active but above $25 \mu \mathrm{g} / \mathrm{mL}$.

\section{EXPERIMENTAL}

Chemistry : All chemicals and solvents used were laboratory grade and directly used. Melting points were determined by open capillary method and are uncorrected. ${ }^{1} \mathrm{H}$ NMR spectra were recorded (in DMSO- $d^{6}, \mathrm{ppm}$ ) on AVANCE-300 MHz spectrometer using TMS as an internal standard ( $\mathrm{s}=$ singlet, $\mathrm{d}=$ doublet, $\mathrm{t}=$ triplet, $\mathrm{m}=$ multiplates and br = brod). Coupling constant $(J)$ are given in $(\mathrm{Hz})$. IR spectra were recorded (in $\mathrm{KBr}$ pallets) on SCHIMADZU spectrophotometer. Mass spectra were recorded on EI-SHIMDZU-GC-MS spectrometer. All reactions and purity of isolated product was monitored by using thin layer chromatography (TLC) using $0.2 \mathrm{~mm}$ silica gel plates 60 F254 (MERCK) and mobile phase petroleum ether and ethyl acetate (80:20). Reaction components were visualized in UV (255 and $365 \mathrm{~nm})$ and iodine chamber.

\subsection{General procedure for Preparation of diethyl (2-phenylquinazolin-4-ylamino) (phenyl) methyl phosphate (4 a-l)}

The mixture of aldehyde $(2.5 \mathrm{mmol}), 2$ - phenylquinazoline-4-amine $(2.5 \mathrm{mmol})$, triethyl phosphite $(3$ $\mathrm{mmol})$ and powdered alum $(20 \mathrm{~mol} \%)$ was stirred magnetically at room temperature. After the completion of reaction as monitored by TLC; $20 \mathrm{~mL}$ ice-cold water was added to the reaction mixture and product was extracted by chloroform $(2 \mathrm{~mL} \times 25 \mathrm{~mL})$. The organic layer washed by brine $(2 \mathrm{~mL} \times 20 \mathrm{~mL})$ and dried over anhydrous sodium sulphate. The solvent was distilled out on Rota-evaporator under reduced pressure to afford the $\alpha$-aminophosphonates which on passing through a column of silica gel $(1.5 \mathrm{gm})$ and elution with petroleum ether-ethyl acetate (7:3) to give pure a-aminophosphonates.

\section{Spectral data of selected compounds}

[1] Yellow crystals: Yield was found to be $95 \%$, m.p. $109-111^{\circ} \mathrm{C}$. IR (KBr) $v \mathrm{~cm}^{-}{ }^{1}: 3281,1228,753 .{ }^{1} \mathrm{H}-$ NMR(DMSO-d $\left.{ }_{6}\right): \square 8.01-7.06(\mathrm{~m}, 14 \mathrm{H}, \mathrm{Ar}-\mathrm{H}), 4.93$ (s, 1H, NH), $4.72(\mathrm{~d}, \mathrm{~J}=24 \mathrm{~Hz}, 1 \mathrm{H}, \mathrm{P}-\mathrm{C}-\mathrm{H}), 3.70-$ $4.21\left(\mathrm{~m}, 4 \mathrm{H}, 2 \times \mathrm{OCH}_{2}\right), 1.32\left(\mathrm{t}, \mathrm{J}=7 \mathrm{~Hz}, 3 \mathrm{H}, \mathrm{P}-\mathrm{O}-\mathrm{CH}_{2}-\mathrm{CH}_{2}\right), 1.14\left(\mathrm{t}, \mathrm{J}=7 \mathrm{~Hz}, 3 \mathrm{H}, \mathrm{P}-\mathrm{O}-\mathrm{CH}_{2}-\mathrm{CH}_{3}\right),{ }^{31} \mathrm{P}-$ NMR (DMSO-d $)_{6}$ ) d 22.61. FAB MS: m/z (\%) $447(\mathrm{M}+\mathrm{K})$, Anal. $\mathrm{C}_{25} \mathrm{H}_{26} \mathrm{~N}_{3} \mathrm{O}_{3} \mathrm{P}$. Calcd: C, 67.12; H, 5.88; N, 9.39, O, 10.75; P, 6.97, Found: C, 67.10; H, 5.86; N, 9.39, O, 10.73; P, 6.92.

[2] Yellow crystals: Yield was found to be $94 \%$, m.p. $178-180{ }^{\mathrm{O}} \mathrm{C} . \mathrm{IR}(\mathrm{KBr}) v \mathrm{~cm}^{-}{ }^{1}: 3281,1228,753 .{ }^{1} \mathrm{H}-$ NMR(DMSO-d $\left.{ }_{6}\right): \square 8.10-7.09(\mathrm{~m}, 13 \mathrm{H}, \mathrm{Ar}-\mathrm{H}), 4.93(\mathrm{~s}, 1 \mathrm{H}, \mathrm{NH}), 4.72(\mathrm{~d}, \mathrm{~J}=24 \mathrm{~Hz}, 1 \mathrm{H}, \mathrm{P}-\mathrm{C}-\mathrm{H}), 3.70-$ $4.21\left(\mathrm{~m}, 4 \mathrm{H}, 2 \times \mathrm{OCH}_{2}\right), 1.32\left(\mathrm{t}, \mathrm{J}=7 \mathrm{~Hz}, 3 \mathrm{H}, \mathrm{P}-\mathrm{O}-\mathrm{CH}_{2}-\mathrm{CH}_{2}\right), 1.14\left(\mathrm{t}, \mathrm{J}=7 \mathrm{~Hz}, 3 \mathrm{H}, \mathrm{P}-\mathrm{O}-\mathrm{CH}_{2}-\mathrm{CH}_{3}\right),{ }^{31} \mathrm{P}-$ NMR (DMSO-d $)_{6}$ ) d 22.61. FAB MS: m/z (\%) $465(\mathrm{M}+\mathrm{K})$, Anal. $\mathrm{C}_{25} \mathrm{H}_{25} \mathrm{FN}_{3} \mathrm{O}_{3} \mathrm{P}$. Calcd: C, 64.53; H, 5.43; F, 4.10; N, 9.06, O, 10.32; P, 6.67, Found: C, 64.51; H, 5.41; F, 4.08; N, 9.03, O, 10.31; P, 6.65.

[3] Yellow crystals: Yield was found to be $90 \%$, m.p. $155-157^{\mathrm{O}} \mathrm{C}$. IR $(\mathrm{KBr}) v \mathrm{~cm}^{-1}: 3281,1228,753 .{ }^{1} \mathrm{H}-$ NMR(DMSO-d $\left.{ }_{6}\right): \square 7.88-6.53(\mathrm{~m}, 13 \mathrm{H}, \mathrm{Ar}-\mathrm{H}), 4.93(\mathrm{~s}, 1 \mathrm{H}, \mathrm{NH}), 5.95(\mathrm{~s}, 1 \mathrm{H}, \mathrm{OH}), 4.72(\mathrm{~d}, \mathrm{~J}=24 \mathrm{~Hz}, 1 \mathrm{H}$, P-C- H), 3.70-4.21(m, 4H, $\left.2 \times \mathrm{OCH}_{2}\right), 1.32\left(\mathrm{t}, \mathrm{J}=7 \mathrm{~Hz}, 3 \mathrm{H}, \mathrm{P}-\mathrm{O}-\mathrm{CH}_{2}-\mathrm{CH}_{2}\right), 1.14$ (t, J = $7 \mathrm{~Hz}, 3 \mathrm{H}, \mathrm{P}-\mathrm{O}-\mathrm{CH}_{2}-$ $\mathrm{CH}_{3}$ ), ${ }^{31} \mathrm{P}-\mathrm{NMR}$ (DMSO-d $\mathrm{d}_{6}$ ): d 22.61. FAB MS: m/z (\%) $465(\mathrm{M}+\mathrm{K})$, Anal. $\mathrm{C}_{25} \mathrm{H}_{26} \mathrm{~N}_{3} \mathrm{O}_{4} \mathrm{P}$. Calcd: C, 64.80; H, 5.67; N, 9.09, O, 10.86; P, 6.69, Found: C, 64.79; H, 5.65; N, 9.07, O, 10.81; P, 6.68.

[4] Yellow crystals: Yield was found to be $89 \%$, m.p. $140-142^{\mathrm{O}} \mathrm{C}$. IR (KBr) $v \mathrm{~cm}^{-1}: 3281,1228,753 .{ }^{1} \mathrm{H}-$ NMR(DMSO-d $\left.{ }_{6}\right): \square 7.88-6.53(\mathrm{~m}, 13 \mathrm{H}, \mathrm{Ar}-\mathrm{H}), 4.93$ (s, 1H, NH), 4.72 (d, J = $\left.24 \mathrm{~Hz}, 1 \mathrm{H}, \mathrm{P}-\mathrm{C}-\mathrm{H}\right), 3.70-$ $4.21\left(\mathrm{~m}, 4 \mathrm{H}, 2 \times \mathrm{OCH}_{2}\right), 1.32\left(\mathrm{t}, \mathrm{J}=7 \mathrm{~Hz}, 3 \mathrm{H}, \mathrm{P}-\mathrm{O}-\mathrm{CH}_{2}-\mathrm{CH}_{2}\right), 1.14\left(\mathrm{t}, \mathrm{J}=7 \mathrm{~Hz}, 3 \mathrm{H}, \mathrm{P}-\mathrm{O}-\mathrm{CH}_{2}-\mathrm{CH}_{3}\right),{ }^{31} \mathrm{P}-$ NMR (DMSO-d $)$ ) d 22.61. FAB MS: m/z (\%) $492(\mathrm{M}+\mathrm{K})$, Anal. $\mathrm{C}_{25} \mathrm{H}_{26} \mathrm{~N}_{4} \mathrm{O}_{5} \mathrm{P}$. Calcd: C, 60.99; H, 5.15; N, 11.39, O, 16.27; P, 6.30, Found: C, 60.97; H, 5.12; N, 11.38, O, 16.24; P, 6.29.

[5] Yellow crystals: Yield was found to be $93 \%$, m.p. $166-168^{\mathrm{O}} \mathrm{C}$. IR $(\mathrm{KBr}) v \mathrm{~cm}^{-}{ }^{1}: 3281,1228,753 .{ }^{1} \mathrm{H}-$ NMR(DMSO-d $)_{6}$ : $\square 7.88-6.53(\mathrm{~m}, 13 \mathrm{H}, \mathrm{Ar}-\mathrm{H}), 2.93\left(\mathrm{~s}, 3 \mathrm{H}, \mathrm{CH}_{3}\right), 4.93,(\mathrm{~s}, 1 \mathrm{H}, \mathrm{NH}), 4.72(\mathrm{~d}, \mathrm{~J}=24 \mathrm{~Hz}$, 1H, P-C- H), 3.70-4.21(m, 4H, 2xOCH $), 1.32\left(\mathrm{t}, \mathrm{J}=7 \mathrm{~Hz}, 3 \mathrm{H}, \mathrm{P}-\mathrm{O}-\mathrm{CH}_{2}-\mathrm{CH}_{2}\right), 1.14$ (t, J = $7 \mathrm{~Hz}, 3 \mathrm{H}, \mathrm{P}-\mathrm{O}-$ $\mathrm{CH}_{2}-\mathrm{CH}_{3}$ ), ${ }^{31} \mathrm{P}-\mathrm{NMR}$ (DMSO-d ${ }_{6}$ ): d 22.61. FAB MS: m/z (\%) $461(\mathrm{M}+\mathrm{K})$, Anal. $\mathrm{C}_{26} \mathrm{H}_{28} \mathrm{~N}_{3} \mathrm{O}_{3} \mathrm{P}$. Calcd: C, 67.69; H, 6.13; N, 9.13, O, 10.42; P, 6.75, Found: C, 67.67; H, 6.12; N, 9.11, O, 10.40; P, 6.71.

[6] Yellow crystals: Yield was found to be $92 \%$, m.p. $168-170^{\circ} \mathrm{C}$. IR (KBr) $v \mathrm{~cm}^{-1}: 3281,1228,753 .{ }^{1} \mathrm{H}-$ NMR(DMSO-d $)_{6}$ : $\square 7.88-6.53(\mathrm{~m}, 13 \mathrm{H}, \mathrm{Ar}-\mathrm{H}), 2.93\left(\mathrm{~s}, 3 \mathrm{H}, \mathrm{CH}_{3}\right), 4.93,(\mathrm{~s}, 1 \mathrm{H}, \mathrm{NH}), 4.72(\mathrm{~d}, \mathrm{~J}=24 \mathrm{~Hz}$, 1H, P-C- H), 3.70-4.21(m, 4H, $\left.2 \times \mathrm{OCH}_{2}\right), 1.32$ (t, J = 7Hz, 3H, P-O-CH $\left.\mathrm{CH}_{2} \mathrm{CH}_{2}\right), 14$ (t, J = $7 \mathrm{~Hz}, 3 \mathrm{H}, \mathrm{P}-\mathrm{O}-$ 
$\mathrm{CH}_{2}-\mathrm{CH}_{3}$ ), ${ }^{31} \mathrm{P}-\mathrm{NMR}\left(\mathrm{DMSO}-\mathrm{d}_{6}\right)$ : d 22.61. FAB MS: m/z (\%) $515(\mathrm{M}+\mathrm{K})$, Anal. $\mathrm{C}_{25} \mathrm{H}_{24} \mathrm{Cl}_{2} \mathrm{~N}_{3} \mathrm{O}_{3} \mathrm{P}$. Calcd: C, 58.17; H, 4.69; Cl, 13.75; N, 8.16, O, 9.32; P, 6.04, Found: C, 58.15; H, 4.68; Cl, 13.73; N, 8.14, O, $9.30 ; \mathrm{P}, 6.00$.

\subsection{Determination of Antimicrobial Activity}

3.3.1. Antibacterial activity : The antibacterial activities of the synthesized compounds 4 a-l were determined by agar diffusion method as recommended by the National Committee for Clinical Laboratory Standards, (NCCLS) [19-21], against selected Gram-positive bacteria viz. Bacillus subtilis (MTCC 441) and Staphylococcus aureus (MTCC 96) and Gram-negative bacteria viz. Pseudomonas aeruginosa (MTCC 1688), and Escherichia coli (MTCC 1650) strains by the agar well diffusion method. Briefly, $0.1 \mathrm{~mL}$ of overnight grown respective bacterial culture was spreaded over the nutrient agar plates. The wells of $6 \mathrm{~mm}$ diameter were prepared on the nutrient agar plates and filled with diluted test compounds separately. For comparison, DMSO and antibiotic Streptomycin were used as a solvent control and as reference antibacterial agent, respectively. Inoculated plates were then incubated at $37^{\circ} \mathrm{C}$ for $24 \mathrm{~h}$ and the resulting zones of inhibition (in mm) were measured. The minimum inhibitory concentrations at which no growth was observed was taken as the MIC value.

3.3.2. Antifungal activity :The compounds were screened for their antifungal activity on the fungal strains Aspergillus niger (MTCC 1789) and Candida albicans (MTCC 227). Fungal suspension (0.1 mL) was spread on Sabourauds agar plates. The wells of $6 \mathrm{~mm}$ diameter were prepared on the inoculated plates and filled with diluted test compounds separately. For comparison, DMSO and antibiotic Nystatin were used as solvent control and reference antifungal agent, respectively. Inoculated plates were then incubated at $30^{\circ} \mathrm{C}$ for $2-3$ days and the resulting zones of inhibition (in $\mathrm{mm}$ ) were measured. The minimum inhibitory concentrations at which no fungal growth observed was recorded as the MIC value.

\section{Figuers and Tables}

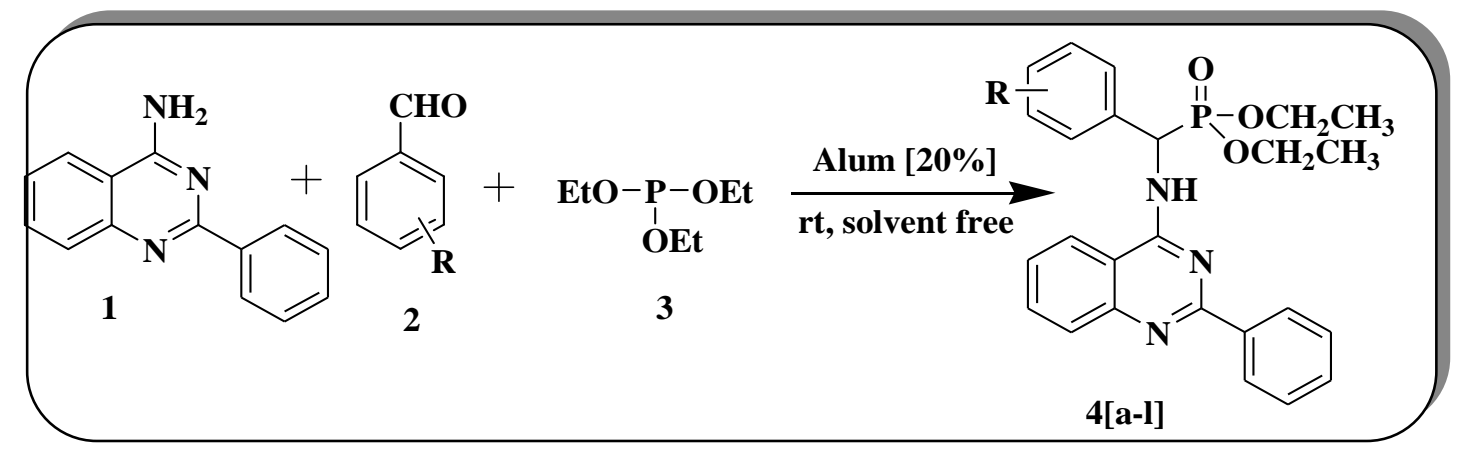

Table: 1 Preparation of diethyl (2-phenylquinazolin-4-ylamino) (phenyl) methyl phosphate 4 [a-l]

\begin{tabular}{|c|c|c|c|c|c|}
\hline Entry & Compound & Aldehyde [R] & Time [Min] & Yield [\%] & M. P. $\left[{ }^{\mathbf{0}} \mathbf{C}\right]$ \\
\hline $\mathbf{1}$ & $4 \mathrm{a}$ & $\mathrm{C}_{6} \mathrm{H}_{5}$ & 10 & 95 & $109-111$ \\
\hline $\mathbf{2}$ & $4 \mathrm{~b}$ & $4-\mathrm{OH}-\mathrm{C}_{6} \mathrm{H}_{4}$ & 15 & 90 & $112-114$ \\
\hline $\mathbf{3}$ & $4 \mathrm{c}$ & $4-\mathrm{Cl}-\mathrm{C}_{6} \mathrm{H}_{4}$ & 12 & 92 & $165-167$ \\
\hline $\mathbf{4}$ & $4 \mathrm{~d}$ & $4-\mathrm{F}-\mathrm{C}_{6} \mathrm{H}_{4}$ & 12 & 94 & $178-180$ \\
\hline $\mathbf{5}$ & $4 \mathrm{e}$ & $2-\mathrm{OH}-\mathrm{C}_{6} \mathrm{H}_{4}$ & 16 & 90 & $112-114$ \\
\hline $\mathbf{6}$ & $4 \mathrm{f}$ & $3-\mathrm{OH}-\mathrm{C}_{6} \mathrm{H}_{4}$ & 15 & 90 & $155-157$ \\
\hline $\mathbf{7}$ & $4 \mathrm{~g}$ & $4-\mathrm{NO}_{2}-\mathrm{C}_{6} \mathrm{H}_{4}$ & 25 & 89 & $145-147$ \\
\hline $\mathbf{8}$ & $4 \mathrm{~h}$ & $3-\mathrm{NO}_{2}-\mathrm{C}_{6} \mathrm{H}_{4}$ & 22 & 89 & $140-142$ \\
\hline $\mathbf{9}$ & $4 \mathrm{i}$ & $4-\mathrm{OCH}_{3}-\mathrm{C}_{6} \mathrm{H}_{4}$ & 15 & 90 & $178-180$ \\
\hline $\mathbf{1 0}$ & $4 \mathrm{j}$ & $4-\mathrm{CH}_{3}-\mathrm{C}_{6} \mathrm{H}_{4}$ & 20 & 93 & $166-168$ \\
\hline $\mathbf{1 1}$ & $4 \mathrm{k}$ & $3,4,5-\mathrm{OCC} \mathrm{H}_{3}-\mathrm{C}_{6} \mathrm{H}_{2}$ & 18 & 90 & $175-177$ \\
\hline $\mathbf{1 2}$ & $4 \mathrm{l}$ & $2,4-\mathrm{Cl}_{-} \mathrm{C}_{6} \mathrm{H}_{3}$ & 15 & 92 & $168-170$ \\
\hline
\end{tabular}


Synthesis, Characterization And Antimicrobial....

Table: 2 Antibacterial Activity of synthesized Compounds (4 a-l)

\begin{tabular}{|c|c|c|c|c|c|c|}
\hline \multirow{3}{*}{$\begin{array}{l}\text { Comp. } \\
\text { No }\end{array}$} & \multicolumn{6}{|c|}{ MIC in $\mu \mathrm{g} / \mathrm{mL}$ [Zone of inhibition, $\mathrm{mm}$ ] } \\
\hline & \multicolumn{4}{|c|}{ Antibacterial activity } & \multicolumn{2}{|c|}{ Antifungal activity } \\
\hline & B. subtilis & S. aureus & E. coli & P. aeruginosa & A. niger & C. albicans \\
\hline $4 \mathbf{a}$ & $25(14)$ & $25(12)$ & $25(15)$ & $25(12)$ & $25(10)$ & $25(14)$ \\
\hline $4 \mathbf{b}$ & $25(6)$ & $25(7)$ & $50(8)$ & $25(10)$ & $25(10)$ & $25(10)$ \\
\hline $4 c$ & $25(15)$ & $25(13)$ & $25(16)$ & $25(15)$ & $25(12)$ & $25(14)$ \\
\hline 4d & $25(13)$ & $25(15)$ & $25(17)$ & $25(12)$ & $25(13)$ & $25(13)$ \\
\hline $4 \mathrm{e}$ & $50(10)$ & $50(14)$ & $50(17)$ & $50(18)$ & $50(16)$ & $50(18)$ \\
\hline 4f & $50(10)$ & $50(14)$ & $50(13)$ & $50(12)$ & $50(14)$ & $50(12)$ \\
\hline $4 g$ & $25(18)$ & $25(14)$ & $25(17)$ & $25(18)$ & $25(16)$ & $25(14)$ \\
\hline $4 h$ & $25(18)$ & $25(14)$ & $25(17)$ & $25(18)$ & $25(16)$ & $25(14)$ \\
\hline $4 i$ & $50(14)$ & $50(16)$ & $50(17)$ & $50(19)$ & $50(13)$ & $50(18)$ \\
\hline $4 \mathbf{j}$ & $50(10)$ & $50(14)$ & $50(17)$ & $50(18)$ & $50(16)$ & $50(18)$ \\
\hline $4 k$ & $25(18)$ & $25(14)$ & $25(17)$ & $25(18)$ & $25(16)$ & $25(14)$ \\
\hline 41 & $25(13)$ & $25(15)$ & $25(17)$ & $25(12)$ & $25(13)$ & $25(13)$ \\
\hline $\begin{array}{l}\text { Streptom } \\
\text { ycin }\end{array}$ & $25(13)$ & $25(15)$ & 25 (17) & $25(12)$ & ND & ND \\
\hline Nystatin & ND & ND & ND & ND & $6.58(18)$ & $25(14)$ \\
\hline
\end{tabular}

\section{CONCLUSION}

In our endeavor, a simple procedure for the synthesis of new a-aminophosphonates $\mathbf{4 a - 4 l}$ in high yields by Kabachnik-Fields reaction using Alum $\left(\mathrm{KAl}\left(\mathrm{SO}_{4}\right)_{2}\right.$ as a catalyst is described. Compound 4 a, 4 c, 4 i found to be potent antibacterial agents against Bacillus subtilis, Escherichia coli and Pseudomonas aeruginosa respectively, where as compound 4 c, $4 \mathbf{f}$ and $4 \mathbf{i}$ shows predominant activity against Aspergillus niger and Candida albicans fungal species. All the synthesized compounds showed moderate antibacterial activity against both gram negative and gram positive bacteria.

Acknowledgments :The authors express their grateful thanks to Principal Dr. N.V.Kalyankar, Yeshwant Mahavidyalaya Nanded for providing laboratory facilities for this work.

\section{REFERENCES}

[1] Barlett, P.A.; Hanson, J.E.; Giannousis, P.P. J. Org. Chem. 1990, 55, 6268

[2] Wang, H.L.; Zhou, J.; Qiu, Y.G.; Feng, K.S.; Chen, R.Y. Phosphorus, Sulfur, Silicon 1995, $104,135$.

[3] (a) Zhou, J.; Chen, R.Y.; Yang, X.F. Heteroatom Chem. 1998, 9, 369. (b) Zhou, J.; Chen, R.Y. J. Chem. Res. (S) 1998, 5, 254. (c) Zhou, J.; Chen, R.Y. Synth. Commun. 1998, 28, 2653.

[4] (a) Kafarski, P.; Lejczak, B. Curr. Med. Chem. Anticancer Agents 2001, 1, 301. (b) Kiran, Y.B.; Reddy, C.D.; Gunasekar, D.; Suresh Reddy, C.; Barbosa, L.C.A.; Annette L. Eur. J. Medicinal Chem. 2008, 43, 885.

[5] De Lombaert, S.; Blanchard, L.; Tan, J.; Sankane, Y.; Berry, C.; Ghai, R.D. Bioorg. Med. Chem. Lett. $1995,5,145$.

[6] Kafarski, P.; Lejczak, B. Phosphorus, Sulfur, Silicon, Relat. Elem. 1991, 63, 193.

[7] Atherton, F.R.; Hassall, C.H.; Lambert, R.W. J. Med. Chem. 1986, 29, 29.

[8] (a) Kukhar, V.P.; Solodenko, V.A. Rus. Chem. Rev (Engl. Trans.) 1987, 56, 859. (b) Yokomatsu, T.; Yoshida, Y.; Shibuya, S. J. Org. Chem. 1994, 59, 7930.

[9] Chandrasekhar, S.; Narsihmulu, Ch.; Shameen Sultana, S.; Saritha, B.; Jayaprakash, S. Synlett. $2003,4,505$.

[10] Takahashi, H.; Yoshioka, M.; Imai, N.; Onimura, K.; Kobayashi, S. Synthesis 1994, 8, 763.

[11] Heydari, A.; Karimian, A.; Ipaktschi, J. Tetrahedron Lett. 1998, 39, 6729.

[12] Azizi, N.; Saidi, M.R. Eur. J. Org. Chem. 2003, 23, 4630.

[13] Chandrasekhar, S.; Prakash, S.J.; Jagadeshwar, V.; Narsihmulu, Ch. Tetrahedron Lett. 2001, $42,5561$.

[14] Ranu, B.C.; Hajra, A.; Jana, U. Org. Lett. 1999, 1, 1141.

[15] Lee, S.; Park, J.H.; Kang, J.; Lee, J.K. Chem. Commun. 2001, 42, 8441.

[16] Akiyama, T.; Sanada, M.; Fuchibe, K. Synlett. 2003, 10, 1463.

[17] Mangte, D. V.; Deshmukh, S. P.; Bhokare, D. D.; Deshpande, A.R. Antibacterial and antifungal activities of some novel thiolactosides. Indian J. Pharm. Sci. 2007, 69, 295-298.

[18] Mangte, D.V.; Deshmukh, S.P.; Bhokare, D.D.; Deshpande Arti, R. Ind. J. Pharma. 2007, 69, 295.

[19] Erturk, O. Biologia 2006, 61, 275

[20] G.H. Shahidi Bonjar, M.H. Fooladi, M.J. Madhavi \& A. Shahgasi, Biotechnology, 3(2), 126 (2004).

[21] L. Jin, B. Song, G. Zhang et al., "Synthesis, X-ray crystallographic analysis, and antitumor activity of N-(benzothiazole-2-yl)-1(fluorophenyl)-O,O-dialkyl- $\alpha$-aminophosphonates," Bioorganic and Medicinal Chemistry Letters, vol. 16, no. 6, pp. 1537-1543, 2006. 